\title{
Análisis histológico de la inserción del fascículo superior del músculo pterigoideo externo al disco articular
}

\author{
Artículo Original
}

\author{
Histological analysis of the insertion of the superior lateral \\ pterigoid muscle fascicle at articular disk
}

Resumen

La inserción del fascículo superior del músculo pterigoideo externo (Pterigoideo Lateral) en el disco articular se presenta a partir del tercio medio de la región central de este y contribuye al desplazamiento del disco y su alteración contribuiría al desplazamiento discal. Sobre la inserción existen diferencias de opinión; unos consideran que solo una pequeńa porción de fibras se inserta en la parte anterior de la cápsula y por ende directa e indirectamente al disco; otros investigadores señalan que se encuentra directamente insertado al disco y al cóndilo o solo en el borde anterior de la cápsula y el disco. El objetivo del estudio fue realizar un análisis histológico de la inserción de este músculo para diferenciar el tipo de fibras que llegan al disco articular (proporción de fibras colágenas y fibras musculares) y determinar el tipo de unión entre el músculo y el disco articular. La muestra de este estudio de tipo transversal y exploratorio estuvo constituido por diesiséis preparados anatómicos de articulaciones temporomandibulares, procesándose para su lectura histológica, con colorantes hematoxilina y eosina, Van Guiesson y Mallory, habiéndose encontrado que la mayor proporción de fibras que llegan a insertarse en el disco articular son colágenas y el tipo de unión entre el musculo y el disco articular es en forma intercalada en forma longitudinal.

Palabras clave: anatomía, histología, músculos pterigoideos, articulación temporomandibular, hematoxilina, eosina

Abstract

The inclusion of the fascicle higher external pterygoid muscle (Lateral Pterigoid) disk articulate occurs from the middle third of the central region and contributes to the displacement of the disc and its alteration help discal displacement. On the insertion, there are differences of opinion; some consider that only a small portion of fiber is inserted into the anterior capsule and thus directly and indirectly to the disk; other researchers point out that it is directly inserted the disk and the condyle or only on the anterior edge of the capsule and the disc. The objective of the study was a histological analysis of the insertion of this muscle to differentiate the type of fibres that reach the disk articulate (proportion of collagen fibers and muscle fibers) and determine the type of union between the muscle and the disc articulate. The sample of this study of transverse and exploratory type, was constituted by prepared diesiseis anatomical joint, temporomandibular, proceding for his histological reading with stains Hematoxylin and Eosin, Van Guiesson and Mallory, having found that the largest proportion of fibers reaching insert disk articulate are collagen and type of Union between the muscle and the disc articulate is sandwiched in a longitudinal way.

Keywords: anatomy, histology, pterygoid muscles, temporomandibular joint, hematoxylin, eosine

\section{Sergio Francisco Alvarado Menacho*,Marieta Petkova Gueorguieva de Rodríguez**, Jorge Eleodoro Villavicencio Gastelú ${ }^{* *}$, Juana Rosa Delgadillo Ávila** , Soledad Ana María Reyes Soto**, Justiniano Sotomayor Camayo**}

\footnotetext{
* Profesor del Departamento Académico de Estomatología Rehabilitadora FO-UNMSM

** Profesor del Departamento Académico de Ciencias Básicas FO-UNMSM

*** Profesor del Departamento Académico Médico Quirúrgico FO-UNMSM
}

Correspondencia:

Mg. Sergio Francisco Alvarado Menacho Departamento de Estomatología Rehabilitadora de la Facultad de Odontología de la Universidad Nacional Mayor de San Marcos

Correo electrónico: salvaradom@unmsm.edu.pe

Fecha de recepción: 24-03-12

Fecha de aceptación: 24-05-12

\section{Introducción}

Es importante tener un conocimiento detallado de los componentes de la articulación temporomandibilar (ATM) y en particular en este estudio el interés es acerca de la anatomía e inserción anterior del fascículo superior del músculo pterigoideo externo con el disco articular, ya que este juega una parte activa en los desplazamientos discales y por lo tanto en la aparición de los trastornos temporomandibulares.

La presente investigación, además del interés académico contribuye directamente en el correcto diagnóstico de las alteraciones de la ATM, e indirectamente en la rehabilitación integral del paciente, específicamente de aquellos que manifiesten dolor articular por cau- sa de la influencia de los desplazamientos discales.

El objetivo del estudio fue realizar un análisis histológico de la inserción de este músculo para diferenciar el tipo de fibras que llegan al disco articular (proporción de fibras colágenas y fibras musculares) y determinar el tipo de unión entre el músculo y el disco articular. 


\section{Material y método}

Esta investigación es de tipo descriptivo, transversal y exploratorio. El estudio se realizó en preparados anatómicos de 8 cadáveres con edad promedio de 25 a 60 años.

La muestra estuvo constituida por 16 preparados correspondientes a la región anatómica de la ATM. (Fig. 1, 2).

\section{Procedimiento del estudio}

Se clasificó y enumeró el material cadavérico.

Se procedió a disecar la región de la ATM, se retiró el arco zigomático para ingresar a la fosa zigomática seccionándo la rama mandibular para exponer y separar el fascículo superior del músculo pterigoideo externo y los componentes de la ATM: cápsula, disco articular, cóndilo temporal y cóndilo mandibular.

Se procedió a su fijación e inclusión en parafina, manteniendo su clasificación inicial

Se efectuó los cortes histológicos y tinción utilizando los colorantes: hematoxilina y eosina (H-E) Fig. 3, 4, y Van Guiesson y Mallory (VG-M) Fig. 5

Se realizó la lectura con microscopio optico con objetivos de 10x, 20x y 40x. y se registró fotográficamente

Se registró lo observado en fichas de recolección de datos y de procedió a su interpretación y análisis.

\section{Resultados}

Se encontró que las fibras que llegan a insertarse al disco articular en mayor proporción son fibras colágenas. Así también que el tipo de unión entre el músculo y el disco articular es en forma intercalada longitudinalmente.

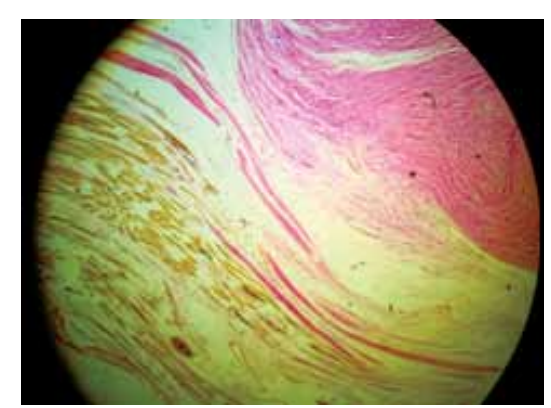

Fig. 4. Corte histológico de disco y músculo pterigoideo externo vientre superior (40x H-E).

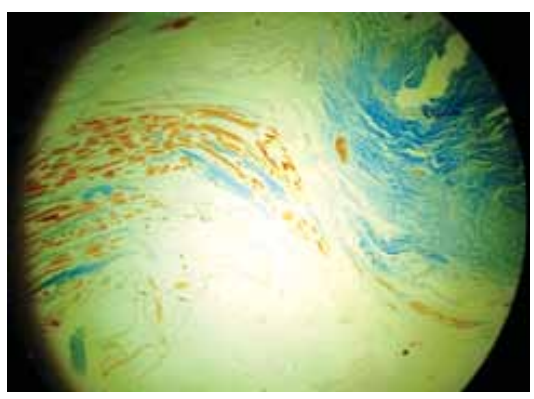

Fig. 5. Corte histológico de disco músculo pterigoideo externo vientre superior (40x VG-M).

\section{Discusión}

La inserción del fascículo superior del músculo pterigoideo externo en el disco articular se presenta a partir del tercio mediano de la región central de este. Se considera que el músculo pterigoideo externo no es un músculo único, se trata de dos músculos distintos con función íntimamente relacionada ${ }^{1}$

La anatomía de la ATM ha sido objeto de debate no solo por su papel en la etiología de la disfunción de la ATM, sino también por el hecho de que el haz superior del pterigoideo externo (Pterigoideo Lateral) se encuentra insertado al disco o al cóndilo de la mandíbula y contribuye al desplazamiento del disco y al dolor articular. $3,4,5,6$

Fig. 3. Corte histológico de disco y músculo pterigoideo externo vientre superior $(10 \mathrm{x} \mathrm{H-E})$. 
Mc Devitt ${ }^{4}$ y Kaplan ${ }^{5}$, pensaron que las fibras superiores del fascículo superior del músculo pterigoideo externo se insertaban en el disco y la cápsula, especialmente en la parte media, mientras que las fibras inferiores se insertaban en el cuello del cóndilo. Sin embargo, existen diferencias de opinión: unos piensan que no se insertan al disco ${ }^{8,9}$, pero una pequeña porción de fibras se encuentran insertadas en la parte anterior de la cápsula y por ende están insertadas directa e indirectamente al disco por sí misma. Otros señalan que el fascículo superior del músculo pterigoideo externo se encuentra directamente insertado al disco y al cóndilo ${ }^{2,10}$. También se cree que solo está insertado en el borde anterior de la cápsula y el disco ${ }^{11}$.

Todos los investigadores están de acuerdo sobre la inserción anterior del fascículo superior del músculo pterigoideo externo al periostio de la fosa infratemporal adyacente al ala mayor del esfenoides. El por qué existe esta confusión no está claro, ya que los estudios involucran tanto exámenes macroscópicos como microscópicos del músculo en relación a la ATM.

En relación a lo anterior, en $1995 \mathrm{He}-$ ylings et al. ${ }^{3}$ realizaron un estudio cuyo propósito era determinar la relación anatómica entre el fascículo superior del músculo pterigoideo externo y el disco, y descubrir si el músculo se inserta directamente en la cápsula y el cóndilo de la mandíbula, además se pretendía determinar cómo se ha desarrollado la confusión en la literatura. Para llevar a cabo esta investigación se emplearon 16 muestras de cadáveres de individuos de 60 a 90 años, 11 de esas muestras eran edéntulos mientras que el resto eran edéntulos parciales; el arco zigomático se removió en todos los casos y se secciono la rama de la mandíbula durante la disección inicial. Los resultados del estudio demostraron que solo una parte de la porción superior del músculo se inserta en la porción anterior de la cápsula, la cual a su vez se encuentra firmemente insertada al disco dando la impresión de que el músculo y el disco están directamente conectados. En todas las muestras se observó que el fascículo superior del músculo pterigoideo externo se inserta en la porción anteromedial de la cápsula, pero presentaban grados variables de inserción a la porción lateral de esta estructura. La parte restante de este músculo se inserta al cóndilo mandibular.
Tener conocimiento detallado de la anatomía del fascículo superior del músculo pterigoideo externo es importante, ya que este juega una parte activa a nivel de los desórdenes temporomandibulares ${ }^{12}$, sin embargo, una revisión preliminar de la literatura reveló la falta de consenso sobre la anatomía de este músculo. De acuerdo con Honee ${ }^{11}$, el pterigoideo externo está formado por una porción superior y una inferior, mientras que otros investigadores como Grant $^{15}$ no identificaron partes separadas. Por otra parte la configuración anatómica de la inserción músculo-disco es debatible. Honee ${ }^{11}$ y Porter $^{4}$ señalaron que el PL se encuentra insertado al disco, mientras que otros encontraron que solo una pequeña fracción de fibras se unía; sin embargo, Pinkert, mencionado por Heylings en su estudio ${ }^{14}$ encontró una septa fibrosa separando al músculo del disco.

\section{Conclusiones}

Los resultados nos muestran que la inserción del vientre superior del músculo pterigoideo externo se manifiesta en el sector anterior del disco articular observándose que llegan a insertarse fibras colágenas en mayor proporción. La unión entre el músculo y el disco articular se presenta en forma intercalada longitudinalmente, lo que podría guiarnos a pensar que el disco si puede ser traccionado directamente por dicho músculo haciéndonos inferir su importancia en los problemas articulares relacionados con los problemas de trastornos témporomandibulares intracapsulares.

\section{Recomendaciones}

Se recomienda realizar estudios en diferentes grupos étnicos y también estudios clínicos para determinar la participación del músculo pterigoideo externo en la etiología de las disfunciones de la ATM.

\section{Referencias bibliográficas}

1. Martins C. M., De Almeida S.M. Estúdio histológico da insercao do músculo pterigóideo lateral na ATM humana. Rev Associação Paulista de Cirurgióes Dentistas (Internet). 2002 set (citado 03 nov. 2008); Disponible en: http:// www.apcd.org.br/Biblioteca/Revista/2002/set_out/338.asp
2. Schmolke C. The relationship between the temporomandibular joint capsule, articular disc and jaw muscles. J Anat. 1994; 184: 335-337.

3. Heylings D, McNeill C. Lateral pterygoid muscle and the temporomandibular disc. J Orofacial Pain. 1999; 9: 9-16.

4. McDevitt W. Functional anatomy of the masticatory system. London: Butterworth. 1989: 560-564.

5. Murray MG, Phanachet I. The human lateral pterygoid muscle: A review of some experimental aspects ans possible clinical relevance. Aust Dent J. 2004; 49: 2-8.

6. Phanachet I, Whittle T. Functional heterogeneity in the superior head of the human lateral pterygoid. J Dent Res. 2003; 82: 106-11.

7. Kaplan A, Assael L. Temporomandibular disorders: Diagnosis and treatment. Philadelphia: WB Saunders. 1991:226-235.

8. Mahan P, Wilkinson T. Superior and inferior bellies of the lateral pterygoid muscle. J Prosthet Dent. 1983; 56: 710.

9. Wilkinson T, Chan E. The anatomic relationship of the insertion of the superior lateral pterygoid muscle in the human temporomandibular joint of the human cadavers. Aus Dent J. 1989; 11:283-288.

10. Le toux G, Duval J. The human temporomandibular joint: Current anatomic and phycologuic status. Surg Radiol Anat. 1989; 11: 283285.

11. Honee G. The anatomy of the lateral pterygoid muscle. Acta Morphol Neerl Scand. 1972; 10: 331-335.

12. Burdi A. Morphogenesis. In: Sarnat B, Laskin D. The temporomandibular joint: A biological basis for clinical practice. ED 4. Philadelphia: WB Saunders. 1992: 36-47.

13. Grant J. A method of anatomy descriptive and deductive. Baltimore: Williams and Wilkins. 1958: 704.

14. White L. The lateral pterygoid muscle: Fact and fictions. J Clin Orthod. 1985; 19: 584-587.

15. Osborn J. The disc of the human temporomandibular joint: Design, function and failure. J Oral Rehabil. 1985; 12: 279 . 